\title{
Statistical analyses of oscillation-effect data within the kindling paradigm
}

\author{
JOHN GAITO and STEPHEN T. GAITO \\ York University, Downsview, Ontario M3J 1P3, Canada
}

\begin{abstract}
Data from a number of kindling experiments involving 60 convulsion trials were evaluated by a truncated principal components factor analysis to determine the number of common factors present. These data were obtained on 123 rats in which periodic low-intensity unilateral stimulation was alternated from one amygdala to the other after six convulsions on each side. Two dependent variables (latency of convulsion, duration of convulsion) were analyzed over Trials 1-60,1-24, 25-60, 1-12, 13-24, 25-36, 37-48, and 49-60 for each dependent variable. Two factors appeared for all latency analyses: primary site stimulation (first side stimulated), secondary site stimulation (second side stimulated). The factor resolution was not clear for the duration measure; two to four factors were suggested in the various analyses.
\end{abstract}

The "kindling effect" has been investigated in a number of laboratories, usually as a model of learning or of epilepsy; however, it is also an example of behavioral change during periodic invariant stimulation that is of interest in its own right (e.g., Gaito \& Gaito, 1974; Goddard, McIntyre, \& Leech, 1969). This event involves a change from normal exploration (Stage 1$\mathrm{NE}$ ), to behavioral automatisms (Stage 2-BA, chewing, eye closure on ipsilateral side, salivation), and finally, to clonic convulsions (Stage 3-CC) in response to electrical stimulation of a specific brain site (e.g., amygdala). Stage 3 behavior has the rat standing on its hind paws while bilateral convulsions of the forelimbs occur. Behavioral, chemical, electrophysiological, and neurological aspects of this effect have been investigated by many researchers (Gaito, 1976b).

In recent research we used a sequence of alternating periodic unilateral stimulations of each amygdala by low-intensity $60-\mathrm{Hz}$ sine waves. Stimulation was imposed on one side until six convulsions had occurred (Phase 1primary site stimulation). Then stimulation was instituted for the contralateral amygdala until six convulsions resulted (Phase 2-secondary site stimulation). This procedure was continued for 10 phases ( 5 for the primary site, 5 for the secondary site). We found consistently that lower values occurred for the primary site, especially in latency data. We have called this effect "the oscillation effect" (Gaito, 1976c) because an "oscillation-type" curve appears over the 10 phases. This effect was present over a number of experimental modifications (e.g., Gaito, 1976a; Gaito, Gaito, \& Nobrega, 1977).

Previously, we performed factor analyses on data from our earlier experiments using the means of the 10 phases for each of the 139 rats as the data for the

This research was supported by a grant from the President's NRC Fund (York University). intercorrelations. Three dependent variables [latency of convulsion, number of trials to six convulsions (criterion), duration of convulsions] were evaluated by a truncated principal components solution. The presence of two factors was suggested in the latency and criterion measures (primary site stimulation and secondary site stimulation) and one in the duration data. A principal components factor analysis over the three dependent variables showed the presence of three factors, those observed in each of the separate analyses. Means of data sometimes can obscure important results that occur with individual data. Thus, in this paper we report the outcome of various statistical analyses with the data from each of the 60 trials.

\section{METHOD}

The data from previous experiments were used. In each experiment, male Wistar rats were implanted bilaterally in the amygdalae with bipolar electrodes. The brain coordinates for electrode implantation were: $.5 \mathrm{~mm}$ posterior to bregma, $4.5 \mathrm{~mm}$ from midline, $8.5 \mathrm{~mm}$ from skull (Gaito \& Gaito, 1974). Seven or more days after surgery, brain stimulation was begun.

The stimulation procedure with most rats was the following. Each was stimulated for $30 \mathrm{sec}$ three times each day by a sine wave of 100-microA intensity from a Lafayette stimulator. After achieving a criterion of 6 clonic convulsions (CCs) with this primary site, stimulation of the contralateral amygdala (secondary site) was begun the next day, using the same procedure, and continued until $6 \mathrm{CCs}$ had occurred. On the following day, stimulation was begun at the primary site again and was maintained until the criterion of $6 \mathrm{CCs}$ was achieved. This alternating procedure continued over 10 of these phases, each involving $6 \mathrm{CCs}$.

Most rats were between 135 and 200 days of age at the beginning of each experiment. However, in one study, aged rats (420 to 475 days of age) were used. Likewise, there were deviations from the usual procedure with a few rats in each experiment. For example, some rats were stimulated one or six times per day; some had 1 or $12 \mathrm{CCs}$ to criterion; some animals received 280-microA and some 560-microA stimulation intensities. 
The data used for the present study were from 123 of the 139 rats that had been stimulated over 10 or more phases of sequential alternation in previous research; 12 subjects had to be dropped because there was only one trial per phase, with the result that these rats had only 10 values available (rather than 60 ). The data for 10 phases were analyzed with two dependent variables: latency (time from onset of stimulation to onset of convulsion) and duration of convulsion (time from onset of forelimb convulsion to complete cessation of convulsion), using the values for each of the 6 trials on each of the 10 phases (60 trials in total). By using the data for each individual trial, the third dependent variable used in the previous factor analyses (criterion: number of trials to $6 \mathrm{CCs}$ ) is lost.

Intercorrelations between the 60 trials for each of the two measures were obtained using the Pearson product-moment correlation procedure. For the factor analyses we used the same procedure as in the previous report (Gaito et al., 1977), a principal components analysis using ones in each diagonal. This allows for $\mathbf{k}$ factors to be extracted if $\mathbf{k}$ variables are involved. However, each successive factor extracts maximum variance, and many of the later of the $k$ factors are trivial. The scree test and characteristic roots $\geqslant 1$ were used as criteria to determine the number of significant factors present. These criteria provide better results than those using a chi-square test of correlation matrix residuals after each factor extraction. The latter procedure tends to overestimate the number of factors (Gorsuch, 1974).

All product-moment correlation coefficients and the statistical manipulations were obtained within the Statistical Package for the Social Sciences, SPSS-10, the University of Pittsburgh, on IBM 370 and DECsystem-10 computers at York University.

\section{RESULTS}

\section{Means}

Figures 1 and 2 show the trend of the means for the 123 subjects over the first 24 trials for the latency and duration measures. The trends for the remaining 36 trials for each measure were similar to those for Trials 13-24. Table 1 contains the means, standard deviations, and linear regression coefficients (for each set of 6 trials) over the 60 trials.

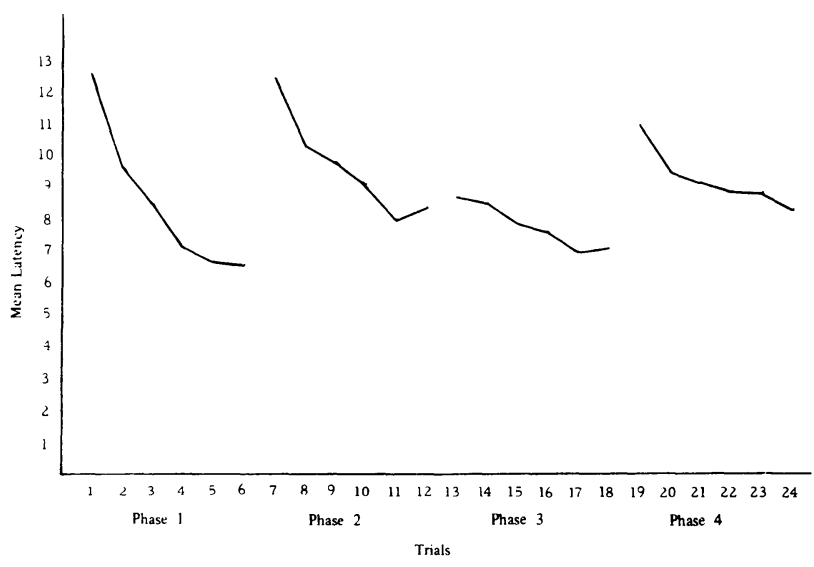

Figure 1. Mean latency (in seconds) over the first 24 trials. Trials 1-6 and 13-18 involve stimulation of the primary site. Stimulation of the secondary site occurs during Trials 7-12 and 19-24.

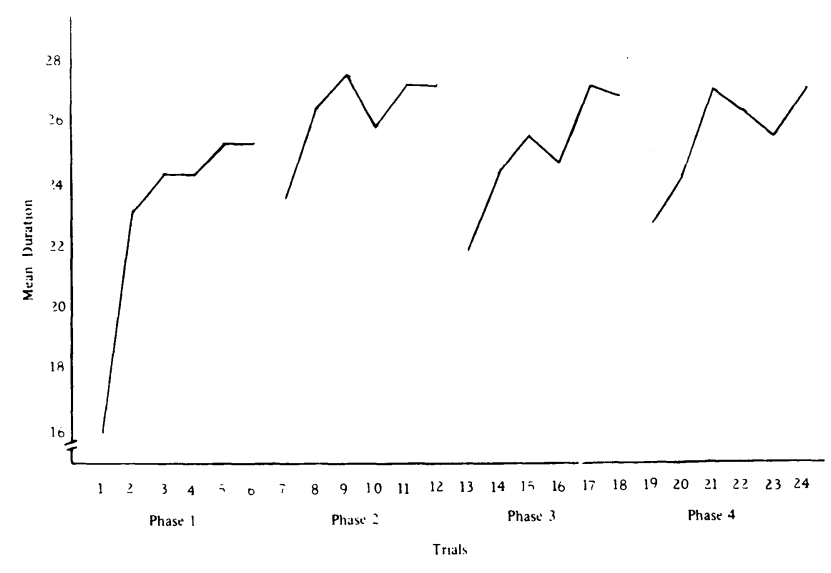

Figure 2. Mean duration (in seconds) over the first 24 trials. Other aspects are as in Figure 1.

At first, the trends of the two measures were the exact opposite of each other. Latency means during the first 12 trials (Phases 1 and 2) indicated a sharp decrement over trials, whereas duration showed a sharp increment. Thereafter, both measures had a more gradual change. By Phases 3 and 4 (Trials 13-24) the pattern for latency had become relatively stable; this aspect was also indicated clearly when the means were analyzed in the previous paper. Relative stability for the duration measure was achieved by Phase 2 .

For the latency data, the means for the secondary site trials $(7-12,19-24$, etc.) were greater than those for the primary site trials $(1-6,13-18$, etc.) over the entire 60 trials. Also, the sets of six primary site trials tended to have lower regression coefficients after the first two phases.

For both measures the first set of six trials was the most variable.

\section{Intercorrelations}

The intercorrelations among the 60 trials were computed. ${ }^{1}$ The important results for latency data were as follows: (1) Coefficients of Trial 1 with each of the other trials in any phase tended to be the lowest. (2) The trials for Phase 1 had the lowest coefficients for between trials within the same phase. (3) By Phase 2 the $r$ values for between trials within the same phase were stable at approximately .60 or greater. (4) The $r$ values for between trials of opposite side phases were low and seldom went above .40. However, if the trials of Phase 1 are excluded, these $r$ values are of a positive nature and tend to be significant. This aspect probably indicates positive transfer over opposite phases.

The results for the duration measure were as follows: (1) The highest rs occurred between adjacent trials, even when one trial involved the primary site and the other, the secondary site. This result is in sharp contrast with the latency measure under these conditions wherein the lowest rs occurred; for the latency data there were sharp breaks between each phase of six trials, with high 
Table 1

Means and Standard Deviations for the 60 Trials and the Linear Regression Coefficients (b) for Each Set of Six Trials

\begin{tabular}{|c|c|c|c|c|c|c|}
\hline \multirow[b]{2}{*}{ Trial } & \multicolumn{3}{|c|}{ Latency } & \multicolumn{3}{|c|}{ Duration } \\
\hline & Mean & SD & b & Mean & SD & b \\
\hline 1 & 12.7 & 8.0 & & 16.0 & 7.6 & \\
\hline 2 & 9.7 & 6.7 & & 23.1 & 11.2 & \\
\hline 3 & 8.5 & 5.8 & & 24.3 & 10.5 & \\
\hline 4 & 7.1 & 5.0 & & 24.3 & 7.6 & \\
\hline 5 & 6.7 & 4.7 & & 25.3 & 7.5 & \\
\hline 6 & 6.6 & 5.6 & -1.17 & 25.3 & 6.8 & 1.52 \\
\hline 7 & 12.5 & 9.3 & & 23.5 & 11.0 & \\
\hline 8 & 10.3 & 7.4 & & 26.4 & 9.4 & \\
\hline 9 & 9.8 & 6.8 & & 27.5 & 9.2 & \\
\hline 10 & 9.1 & 6.8 & & 25.9 & 8.8 & \\
\hline 11 & 8.0 & 5.7 & & 27.2 & 8.0 & \\
\hline 12 & 8.4 & 5.7 & -.80 & 27.2 & 9.3 & .55 \\
\hline 13 & 8.7 & 6.5 & & 21.8 & 8.9 & \\
\hline 14 & 8.5 & 7.0 & & 24.3 & 8.3 & \\
\hline 15 & 7.9 & 6.7 & & 25.5 & 7.6 & \\
\hline 16 & 7.6 & 6.2 & & 24.6 & 7.3 & \\
\hline 17 & 7.0 & 5.9 & & 27.1 & 8.7 & \\
\hline 18 & 7.1 & 5.7 & -.37 & 26.8 & 8.6 & .91 \\
\hline 19 & 11.0 & 7.9 & & 22.6 & 10.8 & \\
\hline 20 & 9.5 & 7.0 & & 24.2 & 10.1 & \\
\hline 21 & 9.1 & 5.9 & & 27.0 & 9.6 & \\
\hline 22 & 8.9 & 5.7 & & 26.4 & 9.6 & \\
\hline 23 & 8.8 & 6.1 & & 25.5 & 8.5 & \\
\hline 24 & 8.3 & 5.3 & -.45 & 27.0 & 9.1 & .72 \\
\hline 25 & 8.5 & 6.4 & & 23.6 & 10.2 & \\
\hline 26 & 8.0 & 6.0 & & 27.2 & 8.7 & \\
\hline 27 & 8.1 & 6.5 & & 26.9 & 10.6 & \\
\hline 28 & 8.0 & 6.0 & & 24.5 & 8.3 & \\
\hline 29 & 7.3 & 5.4 & & 27.3 & 9.1 & \\
\hline 30 & 7.8 & 6.1 & -.16 & 25.9 & 8.3 & .27 \\
\hline 31 & 10.9 & 8.0 & & 22.6 & 11.9 & \\
\hline 32 & 9.2 & 6.1 & & 26.3 & 9.3 & \\
\hline 33 & 9.5 & 6.1 & & 26.3 & 9.6 & \\
\hline 34 & 8.4 & 5.7 & & 27.0 & 9.4 & \\
\hline 35 & 8.2 & 5.4 & & 26.9 & 8.5 & \\
\hline 36 & 8.7 & 5.9 & -.44 & 26.9 & 9.0 & .71 \\
\hline 37 & 9.0 & 6.8 & & 22.4 & 10.9 & \\
\hline 38 & 7.8 & 6.3 & & 26.9 & 11.0 & \\
\hline 39 & 8.0 & 6.1 & & 27.6 & 11.2 & \\
\hline 40 & 7.7 & 5.9 & & 25.8 & 10.2 & \\
\hline 41 & 7.3 & 5.7 & & 26.1 & 9.2 & \\
\hline 42 & 7.7 & 6.3 & -.24 & 26.8 & 9.3 & .51 \\
\hline 43 & 10.1 & 7.2 & & 21.7 & 10.2 & \\
\hline 44 & 9.0 & 6.6 & & 25.6 & 10.3 & \\
\hline 45 & 8.9 & 6.2 & & 25.4 & 10.5 & \\
\hline 46 & 8.7 & 6.4 & & 24.8 & 10.8 & \\
\hline 47 & 8.1 & 5.8 & & 26.5 & 10.7 & \\
\hline 48 & 8.3 & 6.2 & -.34 & 25.8 & 9.1 & .65 \\
\hline 49 & 8.6 & 7.4 & & 21.1 & 9.5 & \\
\hline 50 & 7.2 & 5.8 & & 26.6 & 9.7 & \\
\hline 51 & 7.9 & 6.4 & & 26.1 & 9.1 & \\
\hline 52 & 8.0 & 6.4 & & 24.3 & 9.6 & \\
\hline 53 & 7.4 & 5.9 & & 24.4 & 8.7 & \\
\hline 54 & 7.2 & 5.7 & -.18 & 25.9 & 9.5 & .45 \\
\hline 55 & 9.2 & 7.0 & & 21.8 & 10.4 & \\
\hline 56 & 8.4 & 6.1 & & 26.7 & 11.1 & \\
\hline 57 & 8.6 & 6.9 & & 26.9 & 10.8 & \\
\hline 58 & 8.3 & 6.3 & & 24.7 & 9.9 & \\
\hline 59 & 7.8 & 5.6 & & 25.7 & 10.0 & \\
\hline 60 & 8.0 & 5.7 & -.23 & 26.9 & 9.6 & .58 \\
\hline
\end{tabular}

rs in one phase alternating with low rs in the next phase. (2) Highest rs occurred also between trials within the sets of six for each phase. (3) None of the rs was greater than about .60. This result is also in sharp contrast with latency data, wherein rs of .60 to .80 were common. (4) About half of the rs were nonsignificant or just barely above the .178 required to reject the null hypothesis of zero correlation at a p level of .05 with 123 subjects. More significant rs occurred within latency data.

\section{Factor Analyses}

A principal components solution was used with each measure. Two criteria were used to differentiate significant factors from trivial ones: characteristic roots $\geqslant 1$ and the scree test (Gorsuch, 1974). The latter test involves plotting the characteristic roots, or eigen values, for the various trials. The characteristic roots for trivial factors are in a straight line, whereas those of significant factors show a sharp break or cliff effect. Factor extraction provides an ambiguous solution because the axes are arbitrary. Thus, an Equimax orthogonal rotation procedure was used to provide a solution that is easy to interpret.

These procedures indicate the presence of two factors for the latency measure in the main analysis (Trials 160): primary site stimulation factor and secondary site stimulation factor, which accounted for $67.3 \%$ of the variance. ${ }^{1}$

Factor analyses were performed also on the data for Trials 1-24. 25-60, 1-12, 13-24, 25-36, 37-48, and 49-60. The results were clear. Two factors emerged in each case, the same as for Trials $1-60$, and as in the previous study (Gaito et al., 1977). For most of these analyses the two factors had an angle of approximately $60 \mathrm{deg}$ between them, indicating a correlation of about .50 .

The duration measure did not provide as clear a picture as did the latency data, possibly because many of the rs were nonsignificant. For Trials $1-60$ the two criteria conflicted. There was a suggestion that one, two, or three factors were significant. A one-factor resolution would have been similar to the solution obtained in the previous analysis with means. However, only $21 \%$ of the variance would have been accounted for. Two to four factors were extracted and rotated using an Equimax procedure. Two factors provided the clearest solution, the primary site stimulation factor and the secondary site stimulation factor, as was the case with the latency measure. ${ }^{1}$ However, the plot of these two factors did not show as clear a separation of the trials on the different phases as did the latency data.

The two factors accounted for only $27.4 \%$ of the variance; this aspect is in sharp contrast with the $67.3 \%$ obtained with the latency measure. Also, the communalities (proportion of variance: $\mathrm{h}^{2}$ ) for each trial were very low, varying from .088 to .465 . More than half of these were below .300 . However, $h^{2}$ for Trial 1 was 
not the lowest as in latency data; it was intermediate in magnitude. On the other hand, the latency communalities were sizable; except for the first six trials, these values were .500 or better, with many in the .70 s and some in the low $.80 \mathrm{~s}$. These results indicate that the common factor variance was sizable for the latency trials, but it was almost nonexistent for the duration trials. For the latter measure, a great amount of specificfactor variance and/or error variance was involved.

Three-and four-factor solutions did not improve on the two-factor resolution but tended to cloud the picture.

The duration measure did not provide as clear a picture as did the latency data for the other analyses also. Four factors emerged for Trials 1-24, one for each of the four phases. Three or four factors occurred over Trials 25-60. Two or three factors were suggested in each of the rest of the analyses $(1-12,13-24,25-36$, $37-48,49-60)$. Even when two factors are present, the plot of the two factors did not show a clear separation of the trials on the different phases. Thus, overall the duration measure did not lend itself to a simple and clear factor analysis as did the latency measure.

\section{DISCUSSION}

The latency means tended to become relatively stable after about Trial 12, after one phase with each amygdala, although there was a pronounced tendency for the means of the first trial in all phases to be the greatest one, with a decrease occurring gradually over the remaining trials. An opposite tendency occurred in the duration measure, with lowest values resulting on the first trials and a gradual increment thereafter. Relative stability appeared to occur after the first phase for duration data.

Sharp differences appeared also in the correlation coefficients for the two measures. The pattern of rs for latency were regular, with high rs for those within the same set of six trials and low rs across sets, whereas highest rs resulted from adjacent trials for the duration measure. Likewise, greater rs occurred for latency data than for the duration measure.

Differences between the two measures were prominent also in the factor analyses. The results for the latency measure provided a sharp contrast with the duration analyses with a clear separation of primary site stimulation from that of secondary site stimulation, as occurred in the previous analyses with phase means. Also, the proportion of variance accounted for by the factors extracted and the communalities for each trial were greater for the latency measure. These results are consistent with our observation that the latency measure has been the most sensitive indicator of the oscillation effect and the most important one for our analyses. In fact, even though we record duration data, we do not analyze it in each experiment, because in the first study few examples of oscillation occurred, in sharp contrast with results with the latency measure.

The results of these analyses using individual trials are consistent with the previous results using phase means with latency data in most respects. However, duration results with individual trials provided a less clear picture than was the case with phase means. Obviously, a mean of a set of data can obscure much that is occurring within that set. One aspect that was a probable contributor to the lack of a clear picture was the occurrence of many nonsignificant rs in duration data. Overall, it is gratifying that consistent and clear results are obtained with the measure that has been most important in our research, latency.

In closing it should be noted that these results (as was the case with the previous results) apply to subjects who were exposed to different stimulation conditions, for example, intensities of stimulation, different number of convulsions per phase, and so on. It is obvious that different results might occur if all subjects were treated the same way in all respects. However, these results should provide a possible set of norms over heterogeneous conditions of stimulation.

\section{REFERENCES}

GaI'To, J. The effect of bilateral stimulation during sequential alternation of unilateral amygdaloid stimulation. Bulletin of the Psychonomic Society, 1976, 4, 355-357. (a)

Gaito, J. The kindling effect as a model of epilepsy. Psychological Bulletin, 1976, 83, 1097-1109. (b)

Gaito, J. An oscillation effect during sequential alternations of unilateral amygdaloid stimulations within the kindling paradigm. Physiological Psychology, 1976, 4, 303-306. (c)

Gaito, J., \& Gaito, S. T. Interanimal negative transfer of the kindling effect. Physiological Psychology, 1974, 2, 379-382.

Gaito, J., Gaito, S. T., \& Nobrega, J. N. A factor analysis of data from 10 phases of sequential alternations of amygdaloid stimulation within the kindling paradigm. Physiological Psychology, 1977, 5, 300-310.

Goddard, G. V., McIntyre, D. C., \& Leech, C. K. A permanent change in brain function resulting from daily electrical stimulation. Experimental Neurology, 1969, 25, 295-330.

Gorsuch, R. L. Factor analysis. New York: Saunders, 1974.

\section{NOTE}

1. All intercorrelations through the 60 trials (and factor analysis tables) may be obtained by writing to the first author.

(Received for publication July 25, 1979.) 